\section{AB1080 OPTICAL SPECTRAL TRANSMISSION IMAGING SCORES DECREASE AFTER ONE MONTH OF BIOLOGICAL THERAPY, ESPECIALLY IN RHEUMATOID ARTHRITIS PATIENTS WHO RESPOND TO THERAPY}

\author{
A. Blanken ${ }^{1}$, C. J. Van der Laken ${ }^{2}$, M. Nurmohamed ${ }^{1,2} .{ }^{1}$ Amsterdam \\ Rheumatology and Immunology Center, Location Reade, Amsterdam, \\ Netherlands; ${ }^{2}$ Amsterdam Rheumatology and Immunology Center, Location \\ Amsterdam UMC, Amsterdam, Netherlands
}

Background: Optical spectral transmission imaging (OST) is a new imaging method that measures inflammation in the hands of rheumatoid arthritis (RA) patients. OST might be used to assess disease activity instead of disease activity score 28 (DAS28) or ultrasonography (US). The advantage of OST is that it is fast and not operator dependent. Up to now OST has only been investigated cross-sectionally and it is unknown if and to what extent OST can detect inflammatory changes due to anti-inflammatory treatment for RA.

Objectives: To compare OST measurements before and after 1 month of biological treatment for RA and to compare these OST changes with changes on US and disease activity.

Methods: The HandScan device from Hemics, the Netherlands, was used to measure OST scores for 13 RA patients before and after 1 month of anti-inflammatory therapy. Treatment included tumor necrosis factor inhibitor $(n=10)$, tocilizumab $(n=2)$ and tofacitinib $(n=1)$. OST scores range from 0-66 (one score for both hands) and are based on bilateral wrist, MCP and PIP joints. US was performed in the same joints as OST and semi-quantitatively scored on a scale of 0-3 for grey-scale (GS) synovitis and power Doppler (PD) signal. Joint scores of GS synovitis or PD were summed, resulting is a total GS synovitis score and a total PD score, both also ranging from 0-66. Furthermore, tender joint count 28 (TJC28), swollen joint count 28 (SJC28), DAS28, C-reactive protein (CRP) and erythrocyte sedimentation rate (ESR) were determined. Response to therapy was defined as achieving the minimal clinically interesting improvement of DAS28 (DAS28 difference after 1 month > -1) as proposed by Ward et al. [1]

Results: Baseline OST was $17.73 \pm 6.10$ and this significantly decreased to 16.01 \pm 6.68 (difference $-1.71,95 \% \mathrm{Cl} 0.05-3.38, \mathrm{p}=0.045$ ) after 1 month of therapy This decrease was only present in patients who responded to therapy ( $n=8$; OST decreased from $17.24 \pm 5.98$ to $14.26 \pm 5.65, p=0.01$ ) and not in non-responders $(\mathrm{n}=5$; OST increased from $18.52 \pm 6.90$ to $18.83 \pm 7.87, \mathrm{p}=0.03$ ).

In the total group, also DAS28 (difference $-1.59,95 \% \mathrm{Cl} 0.74-2.45, \mathrm{p}=0.002$ ), SJC28 (difference $4.62,95 \% \mathrm{Cl} 1.50-7.73, \mathrm{p}=0.007$ ), ESR (Wilcoxon Rank $\mathrm{p}=0.008$ ) and CRP (Wilcoxon Rank $\mathrm{p}=0.03$ ) significantly decreased after 1 month of therapy, but TJC28 did not (difference $2.62,95 \% \mathrm{Cl}-2.7-7.91, \mathrm{p}=0.30$ ).

OST change after 1 month of therapy significantly correlated with TCJ28 change (table 1). For GS synovitis the correlation coefficient nearly reached statistical significance. Changes in all other disease activity parameters were not correlated with OST change.

Table 1. Correlation of change in OST measurement with change in disease activity after 1 months of anti-inflammatory therapy

\begin{tabular}{lcc}
\hline & Spearman $r$ & $p$-value \\
\hline Total GS synovitis & 0.54 & 0.06 \\
Total PD & 0.22 & 0.47 \\
DAS28 & 0.35 & 0.25 \\
SJC28 & 0.29 & 0.33 \\
TJC28 & 0.63 & 0.02 \\
ESR & -0.42 & 0.15 \\
CRP & -0.23 & 0.45
\end{tabular}

Conclusion: OST scores significantly decreased after 1 month of anti-inflammatory therapy and only in the RA group that responded well to this therapy. This indicates that OST is capable of detecting therapy induced inflammatory changes in the hands of RA patients. Larger studies are needed to further assess the monitoring value of OST for therapy efficacy in RA patients.

References:

[1] Ward et al. 2015 Clinically important changes in individual and composite measures of rheumatoid arthritis activity: thresholds applicable in clinical trials. Ann Rheum Dis 74(9): p. 1691-6.

Disclosure of Interests: Annelies Blanken: None declared, C.J. van der Laken: None declared, Michael Nurmohamed Grant/research support from: Not related to this research, Consultant of: Not related to this research, Speakers bureau: Not related to this research

DOI: 10.1136/annrheumdis-2020-eular.5811

\section{$\mathrm{AB} 1081$ \\ BLOOD PRESSURE, BMI AND SEX AFFECT OPTICAL SPECTRAL TRANSMISSION IMAGING MEASUREMENTS OF THE HANDS}

A. Blanken ${ }^{1}$, C. J. Van der Laken ${ }^{2}$, M. Nurmohamed ${ }^{1,2} .{ }^{1}$ Amsterdam Rheumatology and Immunology Center, Location Reade, Amsterdam, Netherlands; ${ }^{2}$ Amsterdam Rheumatology and Immunology Center, Location Amsterdam UMC, Amsterdam, Netherlands

Background: Optical spectral transmission imaging (OST) is a new imaging method designed to measure inflammation in the hands of rheumatoid arthritis (RA) patients. The device uses a pressure cuff to occlude venous blood flow, resulting in an increased blood pool in the hands of the patient. In inflamed joints transmission of light through this blood pool is altered due to higher blood content, lower oxygenation and stronger hemodynamics at the location of inflammation [1]. However, altered hemodynamics not related to inflammatory arthritis might also influence OST measurements.

Objectives: In this study we investigated whether hemodynamic and other cardiovascular parameters affect OST measurements in healthy participants.

Methods: OST measurement was done in 37 healthy participants using the HandScan device from Hemics, the Netherlands. Carotid intima media thickness (IMT), pulse wave velocity (PWV) and augmentation index (Alx) were measured with ultrasound and SphygmoCor tonometry. Age, sex, diastolic and systolic blood pressure, pulse, hypertension (defined as systolic blood pressure $>140$ $\mathrm{mmHg}$ or diastolic blood pressure $>90 \mathrm{mmHg}$ or antihypertensive treatment) total cholesterol, $\mathrm{HDL}, \mathrm{LDL}$, total/HDL cholesterol ratio, body mass index (BMI), European SCORE risk assessment, smoking status, cardiovascular history and medication use were collected during the same visit. Associations were investigated using univariate linear regression and multivariate regression adjusting for age and sex.

Results: Participants were $53 \pm 8$ years old and $65 \%$ was female. Systolic and diastolic blood pressure was $129 \pm 19 \mathrm{mmHg}$ and $80 \pm 9 \mathrm{mmHg}$ respectively and $22 \%$ used antihypertensive treatment, $8 \%$ an anticoagulant and $11 \%$ a statin In total $38 \%$ had hypertension and $8 \%$ had a history of cardiovascular disease. Mean OST value was $12.69 \pm 3.47$ and all values were between 5.00 and 20.78 Cardiovascular and hemodynamic parameters that were associated with higher OST measurement were: hypertension $(14.18 \pm 1.12$ versus $11.79 \pm 0.69)$, systolic and diastole blood pressure, BMI and European SCORE (table 1). Also, male sex was associated with a higher OST with and without adjustment for age (15.05 \pm 1.04 versus $11.41 \pm 0.62$ for females)

Table 1. Cardiovascular and hemodynamic parameters associated with higher OST measurement

\begin{tabular}{|c|c|c|c|c|c|c|c|c|}
\hline \multirow[b]{3}{*}{ Hypertension (y/n) } & \multicolumn{4}{|c|}{ Univariate } & \multicolumn{4}{|c|}{ Adjusted for age and sex } \\
\hline & \multirow{2}{*}{$\begin{array}{c}B \\
2.39\end{array}$} & \multicolumn{2}{|c|}{$95 \% \mathrm{Cl}$} & \multirow{2}{*}{$\begin{array}{c}p \\
0.04\end{array}$} & \multirow{2}{*}{$\begin{array}{c}\text { B } \\
2.41\end{array}$} & \multicolumn{2}{|c|}{$95 \% \mathrm{Cl}$} & \multirow{2}{*}{$\begin{array}{c}\mathrm{p} \\
0.02\end{array}$} \\
\hline & & 0.12 & 4.67 & & & 0.37 & 4.45 & \\
\hline Systolic blood pressure & 0.08 & 0.03 & 0.14 & 0.005 & 0.07 & 0.02 & 0.12 & 0.01 \\
\hline Diastolic blood pressure & 0.20 & 0.08 & 0.32 & 0.001 & 0.19 & 0.09 & 0.29 & 0.001 \\
\hline $\mathrm{BMI}$ & 0.24 & -0.01 & 0.48 & 0.06 & 0.26 & 0.05 & 0.47 & 0.02 \\
\hline Sex $(\text { male/female })^{*}$ & 3.67 & 1.56 & 5.78 & 0.001 & 3.54 & 1.29 & 5.79 & 0.003 \\
\hline European SCORE** & 1.24 & 0.09 & 2.39 & 0.04 & $\mathrm{n} / \mathrm{a}$ & & & \\
\hline
\end{tabular}

*Adjusted for age only, ${ }^{\star}$ Not adjusted because age and sex are included in the European SCORE

Other parameters (age, cholesterol, pulse, IMT, having carotid plaque, PWV, Al, cardiovascular history, smoking status and statin, antihypertensive drug and/o anticoagulant use) were not associated with the OST measurement ( $p>0.13$, data not shown)

Excluding the 5 (14\%) participants with hand osteoarthritis showed comparable results, except for $\mathrm{BMI}$ and hypertension. BMI was also significantly associated with OST in the univariate analysis (B $0.24,95 \% \mathrm{Cl} 0.01-0.47, \mathrm{p}=0.039$ ), while hypertension lost its statistical significance (uni B 1.74, 95\% Cl -0.69-4.17, $p=0.15$ multi B $1.44,95 \% \mathrm{Cl}-0.61-3.48, \mathrm{p}=0.16)$.

Conclusion: High blood pressure, BMI, male sex and European SCORE are associated with increased OST values in healthy participants. This indicates that differences in OST measurements of RA patients might not only reflect inflammatory burden but is also dependent on sex, blood pressure and body composition. This should be taken into account when using optical spectral transmission imaging for the assessment of inflammation in RA patients.

References:

[1] Meier et al. 2012 J Biomed Opt 17(8):081420

Disclosure of Interests: Annelies Blanken: None declared, C.J. van der Laken: None declared, Michael Nurmohamed Grant/research support from: Not related to this research, Consultant of: Not related to this research, Speakers bureau: Not related to this research

DOI: 10.1136/annrheumdis-2020-eular.4718 